\title{
Unified platform for M2M Telco Providers
}

\author{
Mário Antunes, João Paulo Barraca, Diogo Gomes, Paulo Oliveira, and \\ Rui L. Aguiar \\ Instituto de telecomunicações, Universidade de Aveiro \\ \{mario.antunes, paulonascimento\}@av.it.pt, \{jpbarraca, dgomes, ruilaa\}@ua.pt
}

\begin{abstract}
Although many environments are powered by M2M solutions, users do not have a simple way to gather their collective knowledge and program devices' behaviour. Also, Telco providers still lack proper components for enabling integrated services over their networks. We present the final architecture of the APOLLO project, which delivers a enhanced M2M platform encompassing sensors, management and applications platform for a major Telco provider. APOLLO builds on top of ETSI M2M specifications and rich service execution environments providing easy orchestration of services to end-users.
\end{abstract}

Keywords: IoT, IoS, Service Orchestration, Telco, M2M

\section{Introduction}

Millions of sensors collect data about equipment status, environmental conditions, process execution, and human activities, presenting huge opportunities to the development of both smart environments and increasingly efficient business processes.

The ETSI M2M standard ${ }^{1}$ is now mature enough to provide solutions for such massive sensing and acting scenarios, and is now being supplemented by the worldwide OneM2M initiative ${ }^{2}$ Although both ETSI M2M and OneM2M provide components and interfaces for low-level communication and management of devices, as well as integration with Telco infrastructures, they do not address the needs for processing and visualisation capabilities for this IoT. As a complement to these approaches, we propose a novel M2M platform that merges the Internet of Things (IoT) with the Internet of Services (IoS), providing the necessary components to create a useful platform for service creation in this environment.

The APOLLO platform combines the ETSI M2M low level communication and management components, with a higher-level data manipulation layer that follows a SOA approach. It provides several services to analyse, process and manipulate sensory data, data routing to multiple tenants, and advanced machine learning processes. Furthermore, it gives users a GUI to design M2M workflows that can be instantiated by the platform. As validation, after being tested in

${ }^{1}$ http://www.etsi.org/technologies-clusters/technologies/m2m

${ }^{2}$ http://www.onem2m.org/ 
two different scenarios, our solution is now being deployed as the reference M2M platform for a major European Telco provider.

The remainder of the paper is organised as follows. The most relevant M2M platforms are discussed in Section 2. In Section 3 we present the conceptual architecture of the APOLLO architecture, and its different domains. The evaluation use cases are described in Section 4 Finally, the conclusions and the future work are presented in Section 5 .

\section{Related Work}

M2M applications and services have been proposed following mostly vertical or limited approaches. This has hindered interoperability and the realisation of a unified M2M platform, able to address multiple use cases.

Sensor Andrew [7 aims to create a multi purpose sensing network. Its primary goal is to have a living laboratory where applications can be rapidly prototyped. The platform enforces the re-utilisation of technology and components, but falls short when compared with modern software approaches as it lacks methods to do preliminary processing of sensory information coming from the gateways, as well as standardised and scalable data store methods for analysing the information collected, hindering future evolution.

SensorAct [3] is a middleware that supports applications performing operations on sensors and actuators, e.g. query current and historical data or trigger notifications. Nevertheless, it fails to provide a strong Application Layer, therefore is not suitable for supporting processing tasks. Furthermore, it does not allow external entities to interact with the devices.

SenseCampus 9] aims to connect places and entities in the physical world with objects in the cyber-space. This coupling creates an ubiquitous service that not only promotes the distribution of information among the various users, but also gives support to activities that take place in the environment.

BuildingDepot 1] provides an extensible and distributed system, enabling storage, access control, and management of information. It's architecture focus on three components: Data Service $($ DataS $)$ stores information generated by sensors together with meta-data that identifies the context and the appropriate sensor; Directory Service links institutions with DataS, and stores meta-data from child DataS to allow searching; User Service stores information on users and groups from a particular institution.

Both SenseCampus and BuildingDepot provide Web access to sensory data, but do not provide any flexible mechanism to process, analyse or transform it. Both projects communicate with the sensors through a non-standard protocol, limiting the addition of third party sensors. Also, they lack a proper management and operational platform with the capability to integrate and audit device operation.

The industry is perhaps even more active than academia in addressing this issue. Xively $\mathrm{b}^{3}$ is an Web based IoT solution, providing management capabilities

\footnotetext{
${ }^{3}$ https://xively.com/
} 
for device provisioning, and creation of communication pipes. Sen.se ${ }^{4}$ is a platform that enables collection, processing and actuation of sensory information. The platform enables users to integrate existing applications based on flows of information. They provide specialised back-ends to disseminate information, but only provide basic data manipulation services. Both solutions lack integration with pre-existing management infrastructures.

Telco operators are interested in providing M2M platforms to their clients, and have building their own M2M portfolio [6]. However, Telco solutions mostly focus in the provision of SIM cards and of basic services around managed connectivity. While useful, these services do not present an environment that fully leverages the existing communication and management capabilities of Telco infrastructures, as well as the user base and communication coverage, a requirement obvious for a Telco approach to M2M provision.

\section{The APOLLO Platform}

In this work we develop a novel IoT/IoS architecture that covers aspects related to network, device management, services and applications overcoming the shortcomings of the solutions before identified. The APOLLO platform aims to enable integration of a wide range of smart devices, both sensing and acting, which are handled by a single, unified platform. Linked to this infrastructure is the existing Telco cellular infrastructure, fully integrated. APOLLO aims is to allow multiple tenants to deploy their services with agility and reduced time to market.

The platform abides to ETSI M2M and can be divided in three major domains: Sensor, Network, and Service (see Fig. 1). These domains closely related with IoT/IoS, enabling the Telco operator to act as the vital glue holding both concepts together, and presenting an offer with added value to clients.

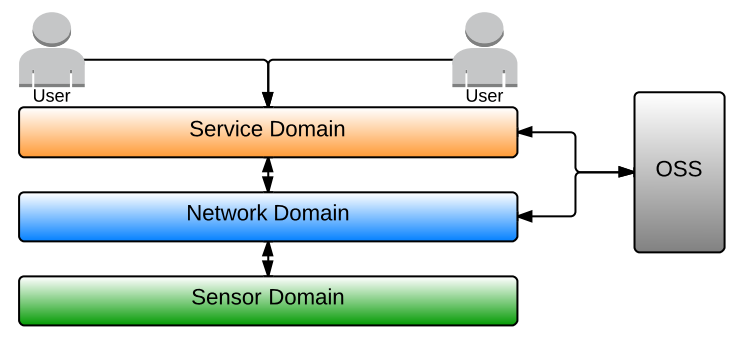

Fig. 1: Architecture of the APOLLO platform.

In the following subsections the several domains are discussed with greater detail.

\footnotetext{
${ }^{4}$ http://open.sen.se/
} 


\subsection{Sensor Domain}

The Sensor Domain (SD) is composed by sensors, actuators, and gateways, that enable integration of physical environments into the management platform. These devices can range from micro-controllers used in low power sensing scenarios, to appliances, cell phones, and other device with M2M capabilities.

Amongst other functions this domain is responsible for enabling smart devices to communicate with the remaining M2M network, abstracting the communication with sensors, and managing the communication facilities at each M2M enabled site through its gateway. Particular scenarios may use different (non ETSI aligned) devices. Still, values are sensed and reported to the upper layers, following strict rules, and using lightweight protocols (see bellow).

APOLLO takes advantage of the ETSI M2M specifications to support seamless integration between heterogeneous sensors and the services present in the upper domains, and supported by a Telco OSS platform. At the level of the Sensor Domain, this reflects in the adoption of a strict architecture for gateways and sensors.

The SD (see Fig. 2 is mostly organised around Service Capability Layers (SCLs). Each SCL is a smart sensor or gateway device, fully supporting the management capabilities of the APOLLO framework. An M2M platform cannot restrict the sensors it supports, as different scenarios and applications will impose very specific operational specifications. Therefore, the APOLLO platform considers the existence of both Smart Sensors, and Legacy Sensors. The first support the full Device SCL (DSCL) architecture and communicate through means of the Gateway SCL (GSCL). The later are not capable of supporting a DSCL component and instead use specialised protocols. Moreover, these sensors can be directly connected to the M2M components or to other broker. When considering hybrid or migration scenarios, where an already existing install base is present, it is common to assume that other brokers may be present.

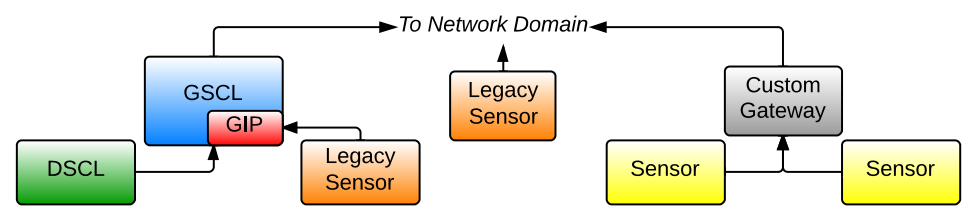

Fig. 2: Components of the Sensor Domain.

We considered that sensors can communicate through standard M2M methods, such as CoAP [8], but also enabled support for other communication solutions in the platform, ranging from proprietary RF signals, to higher level telemetry protocols. Still, the actual payload of each sensor in APOLLO was standardised using JSON [4. For custom sensors that didn't produced JSON documents, adaptation components had the role of creating documents with the correct schema. 


\subsection{Network Domain}

The Network Domain (ND) consists of the device and network management components, hosted by a Telco operator platform. Under the ETSI approach, this reflects the Network Service Capability Layer (NSCL), which in our case is integrated with the existing Operation Support Systems (OSS). The main function of these components is to serve as aggregation points for devices to connect and disseminate information. A relevant aspect is that tenant information must be mapped from the higher layers into the ND. This effectively enables the NSCL to enforce unified access control and accounting, as well as auditing and extended debugging, due to the integration with the Telco OSS (see Fig. 3).

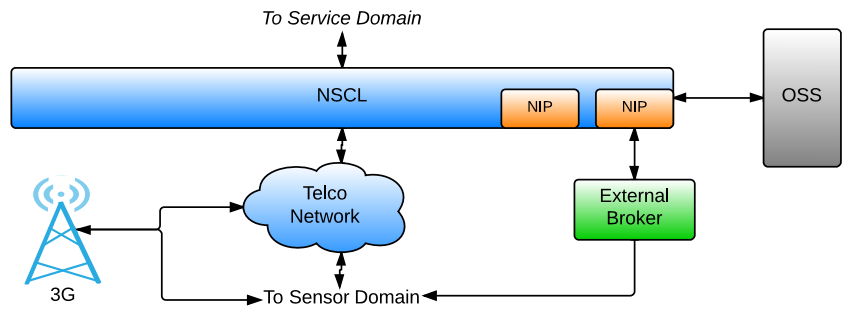

Fig. 3: Components of the Network Domain.

Components can be shared amongst Telco operators/providers as well as by clients with legacy M2M infrastructures. In this case, the NSCL considers the existence of external brokers, which can be directly integrated, or communicate through Network Interworking Proxies (NIP).

As part of an enhanced M2M platform, components of the network domain are also responsible for the management and auditing of devices, providing programmatic interfaces that facilitate device provisioning and debugging. M2M device management is vital as it provides the means for integration of devices, which are heterogeneous by nature and can belong to a multitude of tenants. OMA-DM 5 is our base line for Operations Administration and Management (OAM) support, and can map into each device accordingly to the inherent individual characteristics.

\subsection{Service Domain}

At the Service Domain there is little notion of the device characteristics, and only data objects are exchanged between service endpoints. The components in this domain connect to the Telco OSS and to the NSCL component of the Network Domain, and exchange service information in order to compose a rich SOA environment. It was envisioned that multiple tenants could make use of the M2M platform, while keeping low latency and tenant isolation. Fig. 4 depicts the general architecture of the Service Domain and its most relevant components.

\footnotetext{
${ }^{5}$ http://openmobilealliance.org/about-oma/work-program/device-management/
} 


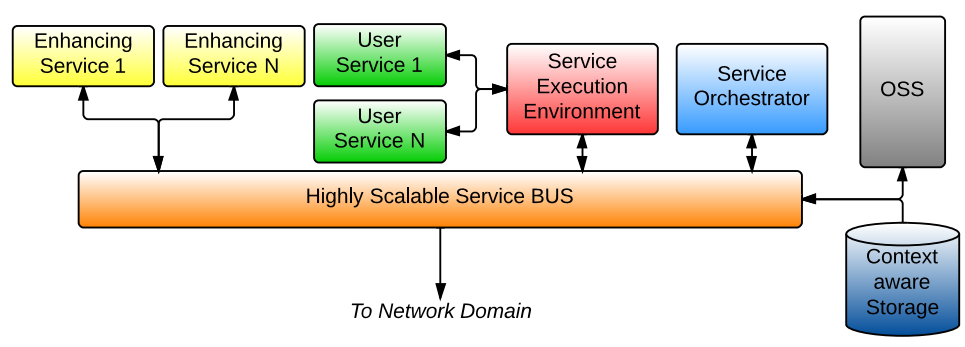

Fig. 4: Components of the Service Domain.

This domain is based on the concept of a Highly Scalable Service Bus (HSSB). An internal component of the bus acts as a Network Application (NA) and registers the currently active topics with the NSCL. Therefore, all information relevant for services and users that reaches the NSCL is injected into the service bus as documents. Usually each context-aware platform defines a context representation that suits their specific needs. This breaks compatibility between platforms and limits the quantity of context information that can be used in M2M applications. To minimize this issue we developed a context storage solution that is agnostic to the context representation and provides advanced search capabilities that overcome the lack of structure [2].

Due to scalability reasons, we consider the service bus actually to be composed by several instances, subscribed to different groups of devices, and with some level of routing between them. From our perspective, as we also consider the existence of multiple NSCLs, the platform can easily be scaled horizontally by adding more instances that deal with a subset of the topics published by sensors. Each HSSB contains multiple Enhancing Services (EN), providing additional functionality over the documents that are published to the HSSB. As an example, an EN can take the temperature, humidity and wind from a Weather Station and enrich the document with the indication that there is a risk of frostbite to plants. Some other ENs can provide richer documents to authorised services-on demand, and effectively play the role of development accelerators and product enhancers, created by Telco providers, to facilitate service development and deployment to their clients.

The platform allows for tenants to develop and deploy services (User Services) directly into it, benefiting from being closer to the data (lower latency). Tenants may deploy two kinds of services: developed on their own following basic web services guidelines and API's, or orchestrated through the supplied graphical user interface. Both service kinds are deployed in the Service Execution Environment (SEE) and made available to all other services through Web Services.

\section{Evaluation Use Cases}

The APOLLO platform was instantiated into multiple scenarios for testing, of which we highlight two: Precision Agriculture focusing in low latency sensing 
and actuation; and Road condition assessment focusing in massive number of events in a Smart City.

\subsection{Smart agriculture}

In the Smart Agriculture scenario we equipped a local agriculture school (ESAC in Coimbra) greenhouse with APOLLO smart sensors and actuators. Sensors where based on low power $\mu \mathrm{C}$, battery/solar powered, capable of monitoring parameters from soil, water, air and radiation. Sensor operation relied on a variable duty cycle, adapted to the power left in their Li-Ion batteries. This was required in order to maintain the network operational in days with reduced solar intensity. Communication between sensors and the gateway used ZigBee radios with mesh capabilities and the CoAP protocol. The GSCL component reported information through a $3 \mathrm{G}$ network. Several Gateway Applications closely interacted with the sensors creating richer information, or enabling low latency direct actuation. In our case, farmers were interested in detecting leaks and avoiding frostbite. Moreover, the flow based service creation interface allowed the definition and analysis of workflows controlling several aspects of the greenhouses. The platform handled about 1 million of events per month, all handled in real time as actuation could be required.

\subsection{Road surface monitoring and pavement analysis}

We targeted also a scenario for road condition assessment through pothole detection, recurring to crowd sourcing, massive data collection, using off-the-shelf mobile devices and machine learning techniques. An Android App was created and made available to citizens who would place their monitoring phones in the dashboard of their cars.

Each monitoring phone would monitor the location, speed, and 3 axis acceleration with a frequency of $15 \mathrm{~Hz}$. The system assesses the road surface condition of several vehicles (use case similar to [5]). Sensors would report information every 5 hours using their $3 \mathrm{G}$ connection, or immediately if a Wifi connection was available. Data flows to an intermediate gateway, and then is dispatched to the NSCL. Finally, information is stored in several databases for the purpose of benchmarking and analysis.

The documents generated by the vehicles are filtered in order to detect high peaks in the $\mathrm{Z}$ (vertical) axis. After we leveraged our cluster based storage for detecting anomalies based on high $\mathrm{Z}$ peaks events, and a machine learning approach for determining anomalies based on a reference road segment. As a result we obtained $82 \%$ in determining potholes under realistic conditions. We had no control over the vehicle, driving style, vehicle condition, or cell phone location. We processed 10 Million reports per month for the duration of the pilot, which enabled us to build a detailed map covering the entire Aveiro region, and even part of the Center region of Portugal. 


\section{Conclusions}

We have presented the architecture of the APOLLO platform. It combines ETSI M2M low level communication and management components with higher-level data manipulation that follows a SOA approach. The platforms allows users to develop innovative M2M services that can be deployed on it. As validation our solution was implemented to be the reference M2M platform for a major Telco provider.

\section{Acknowledgements}

This work has been partially funded by the Portuguese AdI QREN under grant agreements No. 2011/021580 (APOLLO) and CENTRO-07-ST24-FEDER-002031 (CLOUD THINKING).

\section{References}

1. Agarwal, Y., Gupta, R., Komaki, D., Weng, T.: Buildingdepot: an extensible and distributed architecture for building data storage, access and sharing. In: Proceedings of the Fourth ACM Workshop on BuildSys. pp. 64-71 (2012)

2. Antunes, M., Gomes, D., Aguiar, R.: Context storage for m2m scenarios. In: Proceeding of IEEE International Conference on Communications, Selected Areas in Communications Symposium (ICC'14 SAC) (2014)

3. Arjunan, P., Batra, N., Choi, H., Singh, A., Singh, P., Srivastava, M.B.: Sensoract: a privacy and security aware federated middleware for building management. In: Proceedings of the Fourth ACM Workshop on BuildSys. pp. 80-87 (2012)

4. Bray, T.: The JavaScript Object Notation (JSON) Data Interchange Format. RFC 7159 (Proposed Standard) (Mar 2014), http://www.ietf.org/rfc/rfc7159.txt

5. Eriksson, J., Girod, L., Hull, B., Newton, R., Madden, S., Balakrishnan, H.: The pothole patrol: Using a mobile sensor network for road surface monitoring. In: Proceedings of the 6th International Conference on MobiSys. pp. 29-39 (2008)

6. Nkumbwa, R.: Emerging next generation communication technology: Unveiling the ubiquitous society. In: Education and Management Technology (ICEMT), 2010 International Conference on. pp. 1-5 (Nov 2010)

7. Rowe, A., Berges, M.E., Bhatia, G., Goldman, E., Rajkumar, R., Garrett, J.H., Moura, J.M.F., Soibelman, L.: Sensor andrew: Large-scale campus-wide sensing and actuation. IBM Journal of Research and Development 55(1.2), 6:1-6:14 (JanuaryMarch 2011)

8. Shelby, Z., Hartke, K., Bormann, C.: The Constrained Application Protocol (CoAP). RFC 7252 (Proposed Standard) (Jun 2014), http://www.ietf.org/rfc/rfc7252. txt

9. Tokuda, H., Nakazawa, J.: Sensecampus: Sensor enabled cyber-physical coupling for ubiquitous services. Journal of Information Processing 20(1), 45-53 (2012) 